\title{
Caracterización del perfil temporal y de la severidad del delirium postoperatorio en una cohorte de pacientes mayores de 60 años sometidos a cirugía abdominal mayor
}

\author{
Rodrigo Gutiérrez¹,2, Mariana Venegas¹, Isidora Lavado', Antonello Penna²
}

\begin{abstract}
Introducción: El delirium postopetatorio (DPO) es una complicación frecuente, asociada a resultados adversos a largo plazo, como mayor estadía hospitalaria, mayores costos, e incluso mayor mortalidad a largo plazo. Sin embargo, algunos pacientes pueden presentar un cuadro incompleto, y no cumplir con todos los criterios formales de delirium. Esta entidad se conoce como DPO subsindromal (DPOSS), la que también se ha asociado a resultados adversos. A la fecha no se ha evaluado esta entidad en nuestra población, así como tampoco se ha caracterizado su perfil temporal ni su severidad.
\end{abstract}

Objetivo: Caracterizar el perfil temporal, la severidad y la presentación clínica del delirium postoperatorio subsindromal en pacientes mayores de 60 años sometidos a cirugía abdominal mayor bajo anestesia general.

Material y Métodos: Este estudio corresponde a un subanálisis realizado a partir de los datos obtenidos de un estudio prospectivo cuyo objetivo era caracterizar la señal electroencefalográfica intraoperatoria y su eventual asociación con el DPO. Este estudio fue aprobado por el comité de ética local, y se solicitó consentimiento informado a todos los pacientes participantes. Se reclutaron 40 pacientes mayores de 60 años sometidos a cirugía abdominal mayor electiva bajo anestesia general. Posterior a la cirugía, a cada paciente se le aplicó el test CAM (Confussion Assessment Method) para hacer el diagnóstico de DPO (alteración en el dominio aten- ción junto con alteración en el dominio orientación temporoespacial) o DPOSS (alguna alteración en el (AM). Este se realizó dos veces al día por 5 días y fue aplicado por un equipo de terapeutas ocupacionales entrenados. Además, los datos clínicos relevantes durante el período perioperatorio, y hasta el alta, fueron registrados.

Resultados: De los 40 pacientes incluidos, 16 pacientes $(40 \%)$ presentaron alguna alteración en al menos 1 de los CAM aplicados, los que fueron diagnosticados como DPOSS. Nueve pacientes $(56,3 \%)$ debutaron con una alteración en el dominio de la atención. Del total de las alteraciones en los CAM, 35 corresponden al dominio de la atención $(54,7 \%)$ y 29 al dominio de la orientación temporoespacial (45,3\%). En cuanto a la temporalidad del DPOSS, la mitad de los pacientes debutaron las primeras 48 horas. Ningún paciente debutó más allá de la mañana del cuarto día postoperatorio. En relación a la severidad, la mayoría de los pacientes $(68,8 \%)$ presentaron en total menos de 4 alteraciones, y solo dos pacientes $(12,5 \%)$ presentaron más de 8 alteraciones.

Conclusiones: De los pacientes que presentaron DPOSS, la mayoría debutó en la segunda evaluación. Por otra parte, el ítem de atención fue el que se alteró en mayor proporción, y además fue con el debutó la mayoría de los pacientes. Finalmente, la mayor proporción de los pacientes con DPOSS presentan menos de 4 alteraciones en el CAM durante los primeros 5 días postoperatorios. 